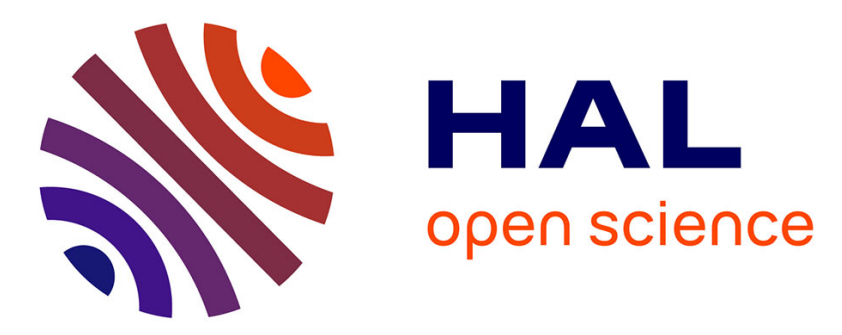

\title{
Analysis of fabrication and crack-induced porosity migration in mixed oxide fuels for sodium fast reactors by the finite element method
}

Tommaso Barani, Isabelle Ramière, Bruno Michel

\section{- To cite this version:}

Tommaso Barani, Isabelle Ramière, Bruno Michel. Analysis of fabrication and crack-induced porosity migration in mixed oxide fuels for sodium fast reactors by the finite element method. Journal of Nuclear Materials, 2022, 558, pp.153341. 10.1016/j.jnucmat.2021.153341 . cea-03581232

\section{HAL Id: cea-03581232 \\ https://hal-cea.archives-ouvertes.fr/cea-03581232}

Submitted on 19 Feb 2022

HAL is a multi-disciplinary open access archive for the deposit and dissemination of scientific research documents, whether they are published or not. The documents may come from teaching and research institutions in France or abroad, or from public or private research centers.
L'archive ouverte pluridisciplinaire HAL, est destinée au dépôt et à la diffusion de documents scientifiques de niveau recherche, publiés ou non, émanant des établissements d'enseignement et de recherche français ou étrangers, des laboratoires publics ou privés. 


\title{
Analysis of fabrication and crack-induced porosity migration in mixed oxide fuels for sodium fast reactors by the finite element method
}

\author{
Tommaso Barani*, Isabelle Ramière, Bruno Michel \\ Commissariat à l'Energie Atomique et aux Energies Alternatives, \\ DES/IRESNE/DEC/SESC/LSC, Saint-Paul-lez-Durance, 13108, France
}

\begin{abstract}
We present an engineering-scale model for the migration of porosity in a fuel pellet experiencing a temperature gradient. The system of coupled pore advection and heat diffusion equations governing the problem is solved through a fixed-point iteration technique. The coupling between porosity and temperature fields is considered via the dependency of pore advection velocity on the local temperature and temperature gradient, and via the dependency of fuel thermal conductivity and of the volumetric power source on the local porosity. We employ the finite element method to discretize the resulting equations. As pure advection solutions obtained by this method are well-known to present spurious spatial oscillations, we introduce stabilization techniques in the pore advection equation. The proposed model is first tested against a benchmark problem representative for the conditions of an uranium-plutonium oxide fuel pellet irradiated in a sodium fast reactor. The results are compared to the those obtained by a model implemented in the BISON fuel performance code. The analysis shows how the results of the newly developed model are in line with those obtained by the reference model, and underlines a superior stability of the solution. The model is then applied to analyze the contribution of as-fabricated and crack-induced porosities in determining the fuel restructuring and in particular the central hole formation. A comparison to experimental data shows the impact of considering crack-induced porosity to predict the extent of the central void.
\end{abstract}

Keywords: Porosity Migration, Temperature gradient, Fixed-point iterations, Finite Element Method, Stabilization Techniques, Mixed Oxide Fuel

\footnotetext{
* Corresponding author

Email addresses: tommaso.barani@cea.fr (Tommaso Barani), isabelle.ramiere@cea.fr (Isabelle Ramière), bruno.michel@cea.fr (Bruno Michel)
} 


\section{Introduction}

The combination of high temperatures and steep temperature gradients in the radial direction of fuel pellets irradiated in light-water or - mainly - fast reactors promotes a substantial restructuring of the as-fabricated microstructure [1]. The main phenomena governing such restructuring are sintering, grain growth, and void/pore migration. Focusing on (mixed) oxide fuel irradiated in fast reactors, such phenomena occur as fuel is brought to power and eventually result in the formation - proceeding from the outer part of the fuel towards the center - of an as-fabricated microstructure zone (i.e., where the temperatures are not high enough to promote the aforementioned phenomena), a zone marked by equiaxed grain growth, a zone marked by columnar grains oriented in the radial direction, and a central void $[1,2]$. Each zone is characterized by a different density (and plutonium content), thus different bulk properties.

To properly analyze the performance of mixed oxide fuels in fast reactors, the phenomena listed above must be represented in the framework of the thermomechanical analysis of the fuel pin. In this work, our attention is drawn on modeling the pore migration mechanism from an engineering-scale perspective, i.e., in the framework of continuum mechanics. The local porosity influences a number of key properties, including fuel thermal conductivity, local power generation, and elastic properties $[1,3,4]$, thus it is a dominant factor in determining the thermal condition in the fuel region.

A consensus arises in the literature about the leading mechanism for pore migration, which is attributed to transport via successive evaporation and condensation of the fuel on the pore surface at different temperatures [1, 2, 5-7]. In detail, the pores are normally filled with low-pressure, low-conducting gas species (e.g., $\mathrm{CO}_{2}$ or $\mathrm{He}$ ), which affects the local temperature gradient. Thus, the presence of the pore modifies the equilibrium partial pressure, which depends on the local matrix composition and temperature, inducing a preferred evaporation of some species from the hot zones and their condensation on the cold one. This transport mechanism, beside being responsible for the migration of the porosity, affects also the redistribution of actinides (namely, plutonium and americium) along the radius, since the chemical species containing these elements are less prone to evaporate and thus accumulates towards the hot pore interface as migration proceeds. For further details about this phenomenon, the reader is referred to $[1,2]$.

In the light of its importance in determining fuel performance of mixed oxide fuels in fast reactor conditions, models have been developed along the years $[1,5-7]$ and included in fuel performance codes to account for porosity migration [8-17]. Recent benchmark exercises [18] underlined the need to ameliorate models on pore migration, showing how the predictions on the central hole size are scattered and not seldom inaccurate. Moreover, in a recent work [19] it was proposed that the displacement of the free volumes due to the porosity migration contributes to the relocation strain of fuel. In particular, a $3 \mathrm{D}$ study realized in this work showed how the mass relocation through the evaporation/condensation along free crack surfaces can lead to a rigid body ra- 
dial relocation displacement of the pellet fragment and then contribute to the closure of the pellet-to-cladding gap.

The aforementioned codes resort either on finite differences/volumes method (e.g., GERMINAL [15] and TRANSURANUS [8]) or on the finite element method (e.g., CEDAR [13] and BISON [14, 17]) to solve the equations governing the migration of porosity. The solution of the (pure) advection equation by the standard Galerkin Finite Element Method (G-FEM), resorting on a centered scheme for the discretization of gradient operator, is known to generate spurious spatial oscillations (see for example [20]). Upwind schemes for the discretization of the gradient operator are known to remove this issue [21], but they are generally not included in finite element libraries frameworks. Instead, in the framework of standard G-FEM resorting on centered schemes, dedicated stabilization techniques - acting as upwind schemes - can be adopted to ensure a stable solution of such family of equations [21, 22].

In this work, we propose an original modeling framework for the coupled solution of the pore migration and heat conduction equations by the finite element method. The open-source library MFEM [23] is used as software platform. The solution of the studied equations requires to solve a non-linear system, whose solution is achieved by a fixed-point iteration algorithm. As for the stabilization techniques, the solution of the pore advection equation is stabilized by two techniques, namely the Streamline-Upwind (SU) and Streamline-Upwind/PetrovGalerkin (SUPG) schemes [22]. A critical comparison to a modern, finiteelement-based code (BISON) is presented and discussed on the example case of as-fabricated porosity migration published in [14].

Since our solver enables an independent or coupled simulation of cracksinduced and as-fabricated porosity migration and, to the best of our knowledge, crack-induced migration has never been accurately simulated at the fuel scale, we propose an analysis of the influence of cracks on the fuel restructuring process. In particular, we present firstly a qualitative assessment of the interaction between as-fabricated and crack-induced porosity, discussing the implications on the central void formation. Finally, we provide a quantitative assessment by analyzing an experiment carried out in the Phenix sodium fast reactor presented in [19] and comparing the results obtained by the presented model against experimental results, underlining the impact of crack-induced porosity on the prediction of the central hole extension. We underline that the focus of the present work is more centered on the development of a physically grounded model describing pore migration and a corresponding consistent mathematical framework to solve its governing equations in the framework of G-FEM. A thorough validation, corroborated by sensitivity and uncertainty studies on the parameters governing the pore migration and temperature distribution, is beyond the scope of the present work and will be the object of future investigations.

The outline of the paper is as follows. In Section 2, we outline the mathematical model developed to represent pore migration. In Section 3, we present some stabilization techniques for the pore advection equations. In Section 4, we present the comparison to the BISON results and critically analyze them. In Section 5, we showcase the results on the crack influence on pore migration 
and fuel restructuring, together with a preliminary assessment of the modeling framework against experimental data. Conclusions are drawn in Section 6 .

\section{Mathematical model}

In this section, we present the equations governing the coupled temperature and porosity fields and the numerical scheme designed to solve the problem.

\subsection{Governing equations and numerical solution scheme}

The equations governing the temperature, $T(\mathbf{x}, t)^{1}$, and porosity, $p(\mathbf{x}, t)$, distribution are the energy conservation (heat conduction) equation and the pore advection equation, respectively, reading

$$
\left\{\begin{aligned}
\rho c_{p} \frac{\partial T}{\partial t}-\nabla \cdot[k(T, p) \nabla T]-q_{v} \frac{1-p}{1-p_{0}} & =0 \\
\frac{\partial p}{\partial t}+\nabla \cdot[\mathbf{v}(T) p] & =0
\end{aligned}\right.
$$

where $\rho\left(\mathrm{kg} \mathrm{m}^{-3}\right)$ is the fuel density, $c_{p}\left(\mathrm{~J} \mathrm{~kg}^{-1} \mathrm{~K}^{-1}\right)$ is the heat capacity, $T(\mathrm{~K})$ is the temperature, $k\left(\mathrm{~W} \mathrm{~m}^{-1} \mathrm{~K}^{-1}\right)$ is the thermal conductivity, $q_{v}\left(\mathrm{~W} \mathrm{~m}^{-3}\right)$ is the volumetric heat source due to fissions, $p_{0}(/)$ is the as-fabricated (initial) porosity, $p(/)$ is the current porosity, $\mathbf{v}\left(\mathrm{m} \mathrm{s}^{-1}\right)$ is the pore velocity. This formulation enforces naturally the respect of the porosity physical bounds between zero (fully dense material) and one (void). In fact, when the porosity approaches one, the heat source is suppressed and therefore the temperature gradient becomes null, in turn suppressing further pore migration, given its dependence on the temperature gradient (pore velocity equal to zero, cf. Eq. (9)). On the other hand, the lower bound for porosity is naturally enforced by the solution of the advection equation itself.

Given the coupled and non-linear nature of the problem, we conceived a fixed-point iteration scheme to achieve system (1) solution. Thanks to the fixed-point algorithm, the heat equation (1a) is linearized evaluating the thermal conductivity at the previous iteration temperature. Therefore, at each iteration, all the equations to be solved are linear (see Figure 1).

At each time step, the convergence check is carried out on the porosity solution, and consists in a mixed relative/absolute criterion, reading

$$
\operatorname{Max}\left(\left|p_{t+1}^{k+1}-p_{t+1}^{k}\right|-\left|p_{t+1}^{k}\right| \cdot \varepsilon_{r e l}-\varepsilon_{a b s}\right)_{\text {Nodes }}<0
$$

where $\varepsilon_{r e l}$ and $\varepsilon_{a b s}$ are the relative and absolute tolerances, respectively. This convergence criterion is preferred to a classical relative error check, since it eliminates the numerical complications arising when the porosity is close to zero and it automatically switches from relative to absolute error when needed.

\footnotetext{
${ }^{1}$ With $\mathbf{x}$ the spatial coordinates and $t$ the time.
} 


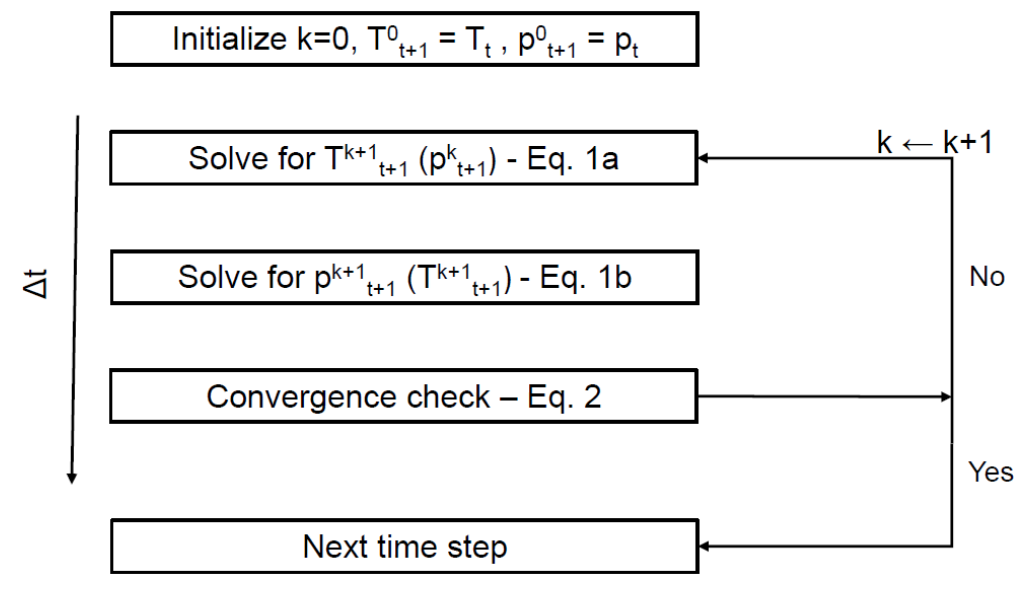

Figure 1: Sketch of the system solution scheme.

The numerical tool we choose to solve Eqs. (1) is MFEM [23], an open source collection of $\mathrm{C}++$ libraries to solve partial differential equations (PDEs) via the finite element method. MFEM allows solving $1 \mathrm{D}, 2 \mathrm{D}$, and $3 \mathrm{D}$ problems using different orders and types of finite elements. Moreover, it allows massive parallelization of the code. Various time integration schemes are available in the MFEM solver, both implicit and explicit.

It is worth underlying that the governing system considered in this work (Eqs. (1)) is conceptually similar to those proposed in previous works on the subject. Sticking to those employing the finite element method, and focusing on the most recent publications $[14,17]$ regarding the BISON fuel performance code, the main difference is found in the pore advection equation and in the numerical strategy to couple energy and pore advection equations.

In fact, in the BISON model a term equal to $(1-p)$ multiplies the pore velocity, representing the suppression of pore migration when the void is formed. Nevertheless, this term does not have a physical ground, i.e., the governing physics is artificially manipulated to suppress pore migration when full "void" is achieved. Indeed, this is an unnecessary constraint, since the temperature gradient naturally vanishes when the porosity equals one, and therefore pore migration is automatically suppressed in this case, because the pore migration velocity is directly proportional to the temperature gradient in the matrix $[1,5-$ 7].

On the numerical aspect, the solution of the governing system in BISON is sought through the Jacobian-Free Newton-Krylov (JFNK) method, which enables a fully-coupled solution of the problem, and considering an implicit time integration, whereas we consider a fixed-point iteration scheme and an explicit time integration. The JFNK method should be regarded, in general, as a leading method to solve coupled non-linear PDEs, in light of its positive sides in terms 
of fast non-linear convergence, scalability, and parallelization possibility [24] For the considered coupled system, which is well-posed, fixed-point iterations converge in few steps. This algorithm can be easily implemented in every computational framework and does not require the estimation of any Jacobian-like matrix, which is generally time consuming to build and computationally expensive to handle. For example, the results presented in Section 4 have been obtained on a personal computer. Finally, for the time integration scheme, implicit schemes theoretically ensure a numerical stability of the solution in time independently from the time step [20]. At the same time, since the physical phenomena occurring in the nuclear fuel might exhibit fast intrinsic dynamics, employing too large time steps would result in inaccurate solutions. Hence, there is not significant differences in time steps that can be employed using explicit and implicit schemes.

\section{Stabilization of the pore advection equation}

Advection-dominated equations solved by the Galerkin Finite Element Method (G-FEM), employing a centered scheme for the discretization of the differential operators, are known to be unstable [20, 22], i.e., to exhibit spurious spatial oscillations in the solution. This problem, classically encountered in other fields such as fluid mechanics, has been encountered also in previous works analyzing the pore migration by the finite element method [14]. In the latter work, a workaround is introduced by adding to the pure advection equation a laplacian term multiplied by a constant diffusivity, which is thought to be representative for pore (bulk) diffusivity. Such a correction is questionable, both on a physical and mathematical perspective. On the one hand, the typical size of fabrication pores (in the micrometric range) is such that surface and bulk diffusion processes are strongly inhibited [25] and could be deemed irrelevant compared to the transport by evaporation/condensation. On the other hand, inserting a constant diffusivity in the advection equation results in a distortion of the solution with respect to the correct one (e.g., [22]).

To overcome the aforementioned problems, one can change the discretization scheme for the gradient (namely, opting for upwind schemes) or use dedicated stabilization techniques for centered schemes. In this work, we implemented in MFEM two classical stabilization techniques for the pore advection equation, namely the Streamline Upwind (SU) and the Streamline Upwind/PetrovGalerkin (SUPG) schemes [22, 26]. These techniques consist in modifying in a consistent manner an advection equation, introducing dedicated stabilization terms and allowing for a stable solution also in the framework of the G-FEM. An interesting observation is that these stabilization techniques share with the upwind discretization of the gradient an essential equivalence, i.e., they result in the addition of a diffusion term to the centered discretization of the advection equation [21]. Employing the SU or SUPG techniques is generally favored, since it allows remaining in the framework of standard finite elements. 


\subsection{Streamline upwind}

The streamline upwind (SU) method consists in adding an artificial diffusion term optimally chosen to balance the G-FEM intrinsic (negative) diffusivity, yielding an exact nodal solution. The idea is to add a diffusivity in the "flow" direction such that the cell Peclet number becomes equal to 1, i.e., only the "useful" amount of diffusivity is introduced. Accordingly, eq. (1b) is modified as follows

$$
\frac{\partial p}{\partial t}+\nabla \cdot(\mathbf{v} p)=\nabla \cdot \overline{\bar{K}} \nabla p
$$

where $\overline{\bar{K}}\left(\mathrm{~m}^{2} \mathrm{~s}^{-1}\right)$ is a second order tensor defined as (e.g., in a bi-dimensional case)

$$
\overline{\bar{K}}=\frac{h^{e}}{2|\mathbf{v}|}\left[\begin{array}{ll}
v_{i} v_{i} & v_{i} v_{j} \\
v_{j} v_{i} & v_{j} v_{j}
\end{array}\right]
$$

where $v_{i}$ and $v_{j}$ are the component of the velocity along the generic $i$ and $j$ direction, $h^{e}$ is the finite element size, and $|v|$ the magnitude of the velocity.

One can notice how this method is consistent, since it depends explicitly on the mesh size, thus approaches zero when the mesh size diminishes. Since it is dependent on the local velocity and on the element size, it introduces the correct amount of diffusivity in the whole domain. It is deemed superior to the approach employed in [14] albeit being practically so simple. It is worthwhile underlying how this approach can be plugged on the advection equation directly in the strong formulation. Nonetheless, for un-stationary problems or stationary problems with non uniform source terms, this stabilization technique is wellknown to be too diffusive, see AppendixA and [22].

\subsection{Streamline upwind/Petrov-Galerkin}

To introduce the SUPG stabilization technique, we pass to the weak form of the advection equation (1b). Being $p$ the generic trial function, $w$ the test function, and $\Omega$ the considered domain, we write

$$
\int_{\Omega} w \frac{\partial p}{\partial t} d \Omega+\int_{\Omega} w \boldsymbol{\nabla} \cdot(\mathbf{v} p) d \Omega=0
$$

Being $\Omega^{e l}$ a partition of the domain, we add to the LHS of the equation above a term of the form

$$
r(p, w)=\sum_{e l=1}^{n_{e l}} \int_{\Omega^{e l}} \mathscr{P}(w)^{e} \tau^{e} \mathscr{R}^{e}(p) d \Omega^{e l}
$$

where $\mathscr{P}(w)$ is an operator applied to the test function and $\mathscr{R}(p)$ the residual of the PDE we are solving. Plugging the aforementioned term in equation (5), 
being the operator $\mathscr{P}(w)$ the skew-symmetric part of the advection operator, we obtain the SUPG formulation ${ }^{2}$

$$
\int_{\Omega^{\prime}} \frac{\partial p}{\partial t}(w+\tau \mathbf{v} \cdot \nabla w)+\int_{\Omega^{\prime}} \boldsymbol{\nabla} \cdot[\mathbf{v} p](w+\tau \mathbf{v} \cdot \nabla w)=0
$$

The upstream parameter $\tau$ is defined as $\tau=h^{e} / 2|\mathbf{v}|$, where $h^{e}$ is the finite element partition size. In the case of a divergence free velocity, the $\mathscr{P}(w)$ operator applied on the divergence term in association with the upstream parameter is similar to the SU formulation. However, it must be underlined that while the SU stabilization can be induced directly in the strong formulation of the advection equation, the full SUPG formulation can be only included in the weak formulation of the problem due to the required modification of the mass matrix. A verification of the implementation of the SUPG method is presented in AppendixA.

\section{Analysis of as-fabricated porosity migration}

To assess the results of the modeling framework exposed above, we present the simulation results on a test-case firstly introduced in [14]. The results are compared to those published in the aforementioned work, showing how the results obtained by our model are in line with those obtained by BISON, yet showcasing a superior stability in the solution thanks to the employed stabilization techniques. In addition, a comparison on the same test-case using the two different stabilization techniques presented above is showed, to demonstrate how the techniques are mostly equivalent on the case of interest.

\subsection{Setup of the calculations}

The computational mesh is a circular sector of radius $2.675 \mathrm{~mm}$ spanning $\pi / 8$ in the angular direction. We considered meshes, especially for the comparison to the BISON results, having different densities, namely 50 and 100 intervals in the radial direction. Non-conforming meshes are employed in this section, trying to preserve an aspect ratio close to the unity of the elements, which are of quadrilateral, first order type. The oxygen-to-metal ratio is taken equal to 1.975 and the plutonium content equal to $20 \mathrm{wt} . \%$.

For the heat conduction equation, a uniform initial temperature equal to 623 $\mathrm{K}$ is considered. A time-varying Dirichlet boundary condition is applied on the outer surface, linearly varying from 623 to $1300 \mathrm{~K}$ over a time period of $10^{4} \mathrm{~s}$. Over the same time, the linear power is brought to $500 \mathrm{~W} \mathrm{~cm}^{-1}$. A zero flux

${ }^{2}$ From there on, we will employ the notation

$$
\int_{\Omega^{\prime}}=\sum_{e l=1}^{n_{e l}} \int_{\Omega^{e l}}
$$


boundary condition (homogeneous Neumann boundary condition) is enforced at the other surfaces, enabling axisymmetry.

As for the pore advection equation, a uniform initial condition with a porosity equal to 0.15 is set. The boundary conditions enforced in this equation are tricky. In fact, the pure advection equation does not induce "physically" sounding natural boundary conditions and its solution normally involves enforcing Dirichlet BC at the "inlet" of the domain [22]. On the other hand, adding a stabilization technique such the ones introduced in Section 3 induces a "diffusive" flux natural boundary condition, which can be more easily justified from a physical perspective. Considering the SU stabilization technique, the naturallyinduced weak boundary condition has the form of a homogeneous Neumann boundary condition

$$
\int_{\Gamma} w[(\overline{\bar{K}} \nabla p) \cdot \mathbf{n}] d \Gamma=0
$$

which takes into account both velocity (through the diffusion-like coefficient) and normal porosity gradient, while the latter is the only term considered in [14].

The relative and absolute tolerances in the fixed-point iteration scheme are set to $10^{-6}$ and $10^{-8}$, respectively. The time integration was carried out considering a time step of $1 \mathrm{~s}$ and an explicit forward Euler time integration scheme. The respect on the Courant cell condition (i.e., $C o=\sum_{i=1}^{N} \frac{v_{i} \Delta t}{\Delta x_{i}}<1$ for every cell) is controlled at each time step, since the velocity varies with time.

\subsection{Model parameters}

The pore velocity expression is known to be a very uncertain yet pivotal property for the assessment of pore migration [27]. Different correlations have been developed in the years, for both $\mathrm{UO}_{2}$ and $(\mathrm{U}, \mathrm{Pu}) \mathrm{O}_{2}[1,5-7,28-31]$, whose results showcase a substantial scattering [27]. More sophisticated approaches, taking into account the vapor pressures of the different species found in the vapor phase, are illustrated in [15, 17].

In this work, the pore velocity is evaluated according to Sens [7], as was done already in a previous work on the subject [14], reading

$$
|\mathbf{v}|=c_{0}\left(c_{1}+c_{2} T+c_{3} T^{2}+c_{4} T^{3}\right) \Delta H_{s} P_{0, s} \exp \left\{-\frac{\Delta H_{s}}{R T}\right\} T^{-2.5}|\nabla T|
$$

where $c_{0}, c_{1}, c_{2}, c_{3}$, and $c_{4}$ are constants, $\Delta H_{s}\left(\mathrm{~J} \mathrm{~mol}^{-1}\right)$ is the heat of vaporization, $P_{0, s}$ is a material parameter, and $R\left(\mathrm{~J} \mathrm{~mol}^{-1} \mathrm{~K}^{-1}\right)$ is the universal gas constant.

The pore velocity depends in principle on the temperature gradient across the pore itself, but a relationship to the temperature gradient across the matrix has been classically considered in the literature, to couple the solution of the heat conduction equation directly to the pore advection equation. In this way, when the central hole is formed and the heat generation is suppressed (cf. equation (1a)), the gradient flattens and naturally suppresses the pore advection. 
The thermal conductivity is accounted for considering the correlation proposed by Kato and coworkers [32], discarding the correction terms accounting for Am and $\mathrm{Np}$ contents (since we are not investigating minor actinides bearing $\left.(\mathrm{U}, \mathrm{Pu}) \mathrm{O}_{2}\right)$ and replacing the porosity correction term by the Maxwell-Eucken model [33], which is a more appropriate way to calculate the thermal conductivity of a two-species mixture as it is modeled in this case ${ }^{3}$. Thus, the Kato correlation for the temperature-dependent part reads

$$
\begin{aligned}
& k(T)=\frac{1}{\left(2.713 \cdot x+1.595 \times 10^{-2}\right)+(2.493-2.625 \cdot x) \times 10^{-4} \cdot T} \\
& +\frac{1.541 \times 10^{11}}{T^{5 / 2}} \exp \left(-\frac{1.522 \times 10^{4}}{T}\right)
\end{aligned}
$$

whereas the complete correlation reads

$$
k(T, p)=k(T) \frac{k_{H e}+2 k(T)-2 p\left(k(T)-k_{H e}\right)}{k_{H e}+2 k(T)+p\left(k(T)-k_{H e}\right)}
$$

where $x(/)$ is the deviation from stoichiometry and $k_{H e}\left(\mathrm{~W} \mathrm{~m}^{-1} \mathrm{~K}^{-1}\right)$ is the thermal conductivity of the pores. As far as the thermal conductivity calculation is concerned, we assume the pore to be filled with helium, for which we take a representative, constant value, equal to $0.69 \mathrm{~W} \mathrm{~m}^{-1} \mathrm{~K}^{-1}$. The correction of thermal conductivity on burnup is not considered in the present study, since we are analyzing only very short irradiation histories. A modification of Eq. (11) to account for burnup would be straightforward and could rely, for example, on the recent work published by Magni and coworkers [34]. It is worth noticing that redistribution of plutonium would occur and influence the local thermal conductivity (directly and affecting the oxygen-to-metal ratio), but we are not accounting for this phenomena in the proposed model since we focus more on providing a robust and consistent modeling of pore migration, rather than a comprehensive fuel behavior module.

\subsection{Comparison of the $S U$ and $S U P G$ techniques}

The results obtained on the test-case employing the SU and SUPG stabilization techniques are reported in Figure 2. Two different mesh densities are considered, namely 50 (Fig. 2a) and 100 (Fig. 2b) elements.

The results are in line with theoretical expectations. We can see how the spurious oscillations classically encountered when solving the advection equation by the G-FEM are removed by both techniques. In fact, the difference between the two techniques is minimal, with the results obtained by the SU method being slightly more diffusive than those obtained by the SUPG method. Substantial differences between these techniques arise when the initial condition is

\footnotetext{
${ }^{3}$ Other approaches for the porosity correction of the thermal conductivity are available in the open literature (e.g., see [4]). Indeed, the correction employed here allows to directly pass from the conductivity of pure oxide to the conductivity of pure gas (helium) when the central hole forms, without the need of introducing step-wise thresholds.
} 


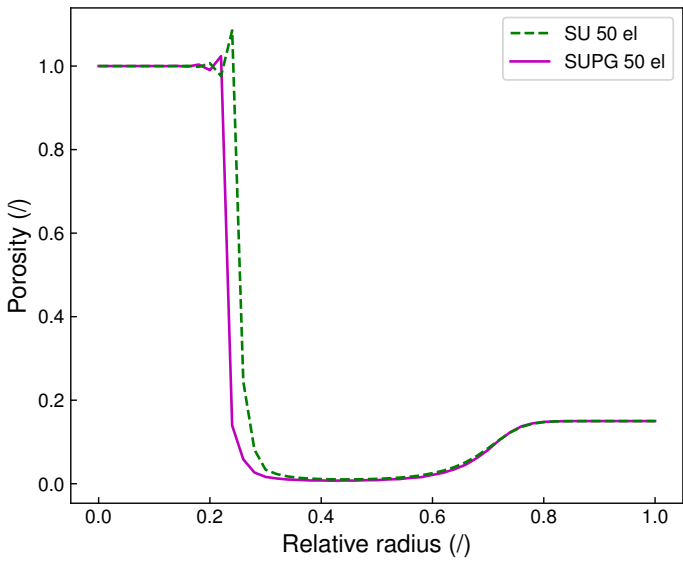

(a) 50 elements

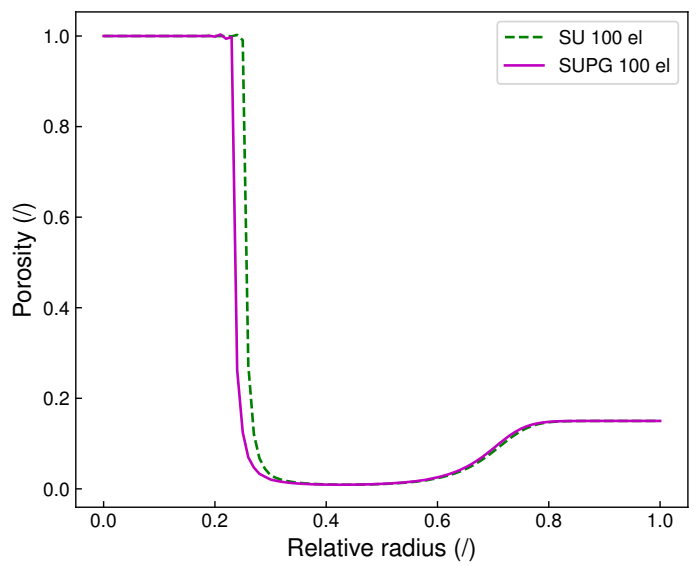

(b) 100 elements

Figure 2: Comparison of the results in terms of porosity as a function of the local radius obtained with MFEM and considering the SUPG and SU stabilization techniques, using respectively 50 (Figure 2a) and 100 element (Figure 2b) meshes. The overshooting of the porosity with respect to its physical bound (i.e., the peak greater than one) employing the coarser mesh (Figure 2a) is a numerical artifact. In fact, it is a result of the steep gradient of the pore velocity across a single mesh element and its discretization in the framework of G-FEM.

The few remaining oscillations found where the porosity is subject to a very steep variation can be mitigated by the mesh refinement. Indeed, they will always appear due to (i) the element-wise, steep gradient of the pore velocity and given that G-FEM relies on the support of the test functions taking into account all neighboring nodal contributions, and (ii) due to the non divergencefree nature of the physical problem.

\subsection{Analysis of calculation results}

The SU stabilization is the technique chosen to obtain the results presented in this work, if not stated otherwise. The choice is due to the fact that, as mentioned above, the results obtained by SU and SUPG techniques are very close for the problem of interest, with both the techniques successfully removing the spurious oscillations in the solution of the pore advection equation. Moreover, 
as can be seen from the mathematical formulation, the SU method does not modify the mass matrix of the associated algebraic problem, whereas the SUPG modifies it (the time derivative of the porosity is multiplied by the gradient of the test function). The results herein presented are obtained employing a computational mesh having 100 radial elements, and considering an explicit Euler (forward Euler) time integration scheme.

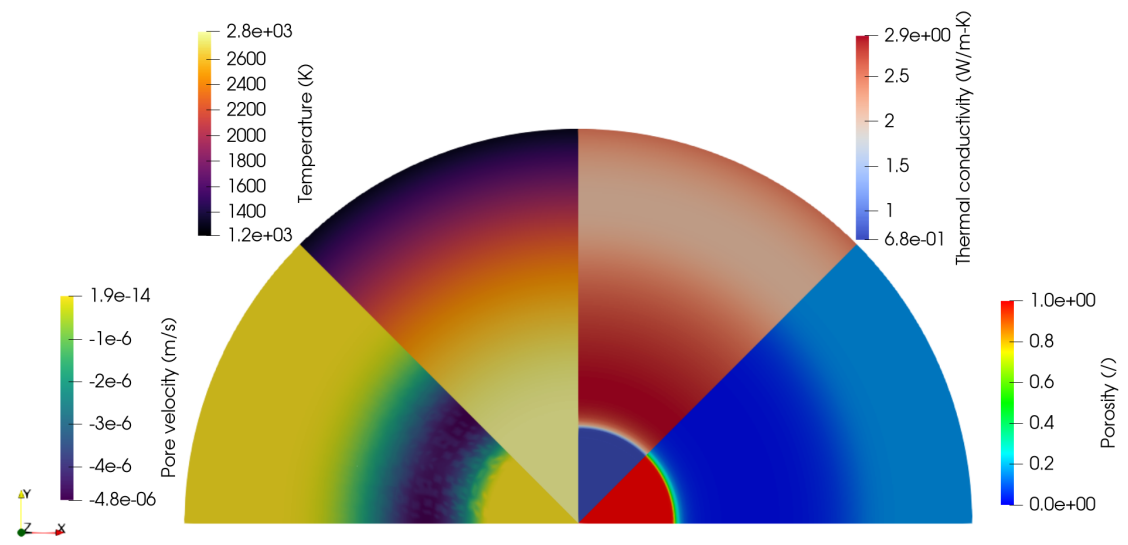

Figure 3: Anti-clockwise, contour plots of porosity, thermal conductivity, temperature, and pore velocity (radial component) after $10^{4} \mathrm{~s}$. The various interdependences can be appreciated.

Figure 3 reports a collection of the results obtained at the end of the considered time period, highlighting some of the main quantities governing the problem. Detail analyses of such quantities are reported in Figures 4 and 5. The coupled nature of the variables and the parameters naturally arises in such results. For example, in Figure 4 the pore advection velocity is reported, together with the two quantities mostly governing it, the temperature and the temperature gradient. It can be appreciated that up to 0.2 relative radius, the temperature gradient is null (since the porosity is equal to one and there is not heat generation), and this is suppressing the pore migration. Thermal conductivity of the pore/oxide "mixture" and its dependencies on porosity and temperature can be glimpsed in Figure 5. It can be noticed the synergic effect of porosity and temperature on such a property: in fact, the maximum values are reached in the outer part, when the temperature is low enough to dominate the effect of the local porosity and allow for an efficient transport mechanism, and around 0.3 relative radius, where the almost absence of porosity due to its migration and the high temperatures result in a high conductivity. Moreover, where the porosity reaches one, i.e., in the central void, the constant value of the thermal conductivity corresponds to the helium conductivity. It is worth underlining that we do not include in this analysis the plutonium redistribution [1, 2, 27], which would surely affect the radial power profile, hence the 


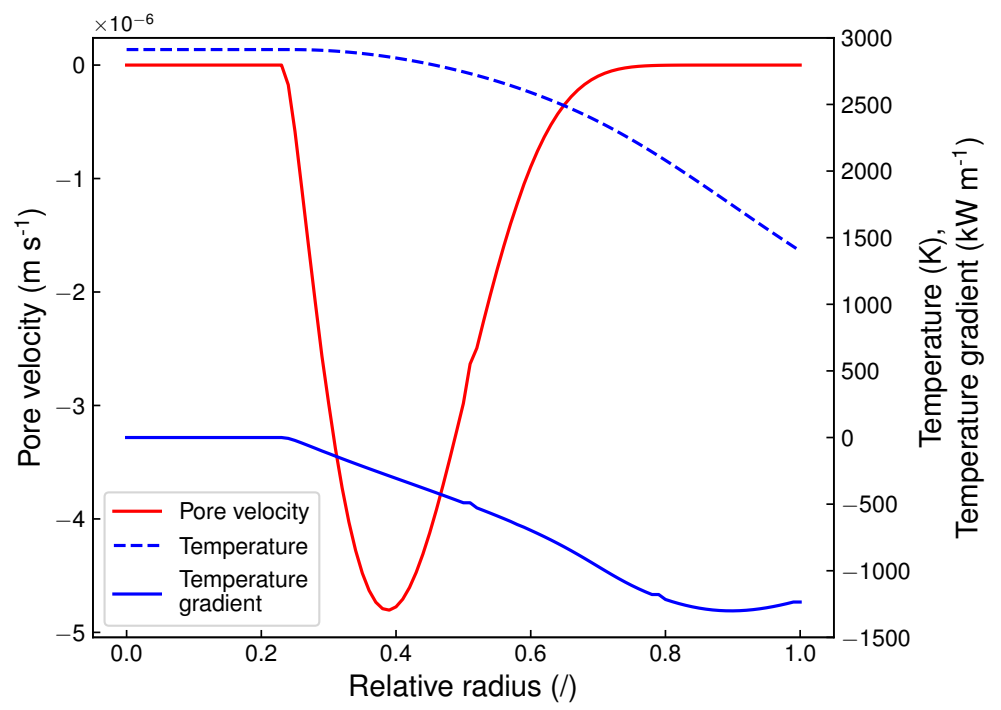

Figure 4: Pore velocity (component in the radial direction), temperature and temperature gradient as a function of the relative radius.

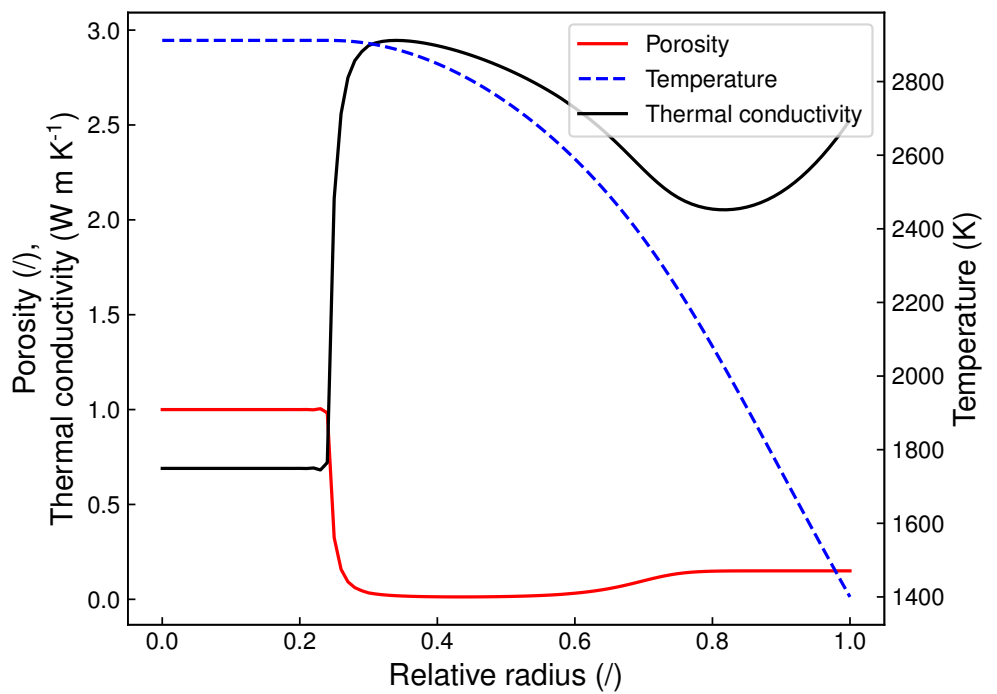

Figure 5: Thermal conductivity, porosity, and temperature as a function of the relative radial position at the end of the test-case. 


\subsection{Comparison to the BISON calculations}

The result of the presented model have been compared to those presented in [14] obtained using BISON. In particular, we are interested in comparing the results on the porosity obtained with different mesh densities.

The solutions are compared in Figure 6. As mentioned above, the SU stabilization is employed in this section. Overall we can see that the solutions are in good agreement. Indeed, we can underline how the solutions obtained using the coarsest mesh (50 elements), showed in Figure 6a shows different degrees of stability, the one obtained with our approach demonstrating a superior stability, in both the void and the restructuring zone. As discussed in Section 4.3, the little oscillations observed in our results near the interface between void and bulk are inherently due to the G-FEM formulation. Let us mention that these oscillations remain substantially constant in time and follow the interface void/bulk.

Our approach surpasses the one reported in [14] for two main reasons. First, we employ a mathematically consistent stabilization technique, based on the SU method, whereas in BISON a constant diffusivity is included, to change the PDE nature from hyperbolic to elliptic and to limit the spurious oscillations in the solution of the pore advection equation. This approach, which resembles the SU formulation from a practical perspective, is not consistent (i.e., it does not vanish when the mesh size tends to zero) from the finite element perspective and needs to be tuned based on the user experience. Second, we solve for a more correct equation governing the pore advection, not including the term $(1-p)$ as done in the BISON formulation [14, 17], which the authors claim to be needed to suppress pore advection when the porosity approaches zero. In fact, the coupling between the pore advection and energy equation with the expression of the parameters as reported, together with a proper stabilization of the solution, is guaranteeing the observation of the physical porosity limit. We postulate that this term is responsible for the difference between our solution and the one obtained by the BISON code, since the velocity magnitude is multiplied by a factor smaller than one, thus "reducing the migration" of porosity with respect to the case where it is not considered (as in our formulation).

\section{Analysis of crack-induced porosity migration}

The role played by cracks as a source of lenticular pore has been outlined by several authors in the literature [2, 7], despite no models elucidating the physical mechanism are available at the moment. In a recent work [19], such mechanism was assumed to play a substantial role in the relocation of fuel at beginning of life. In addition, the healing of cracks by distillation of heavy metal components has been theorized and observed in $(\mathrm{U}, \mathrm{Pu}) \mathrm{O}_{2}[35,36]$. In this section, we present a qualitative analysis on the interaction of as-fabricated and crack-induced porosity, to draw more general conclusions on their impact on fast reactor pellet performance. Moreover, we present a preliminary assessment of the model by comparing its predictions to an experimental results relative to a 


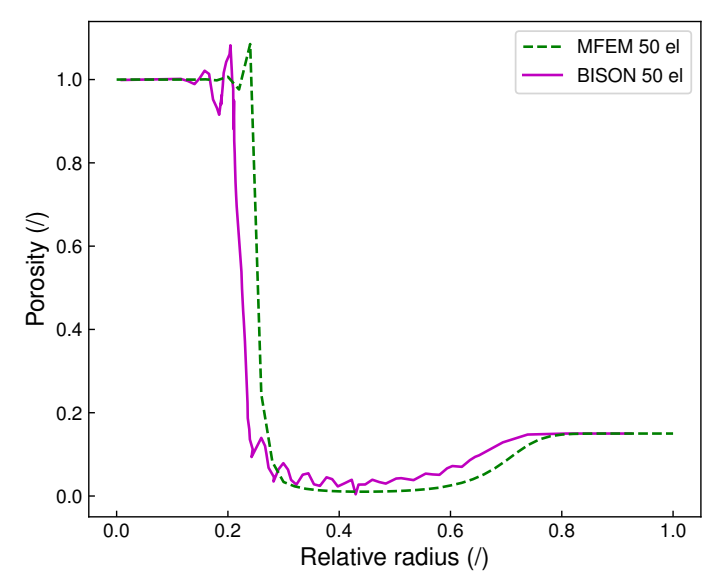

(a) 50 elements

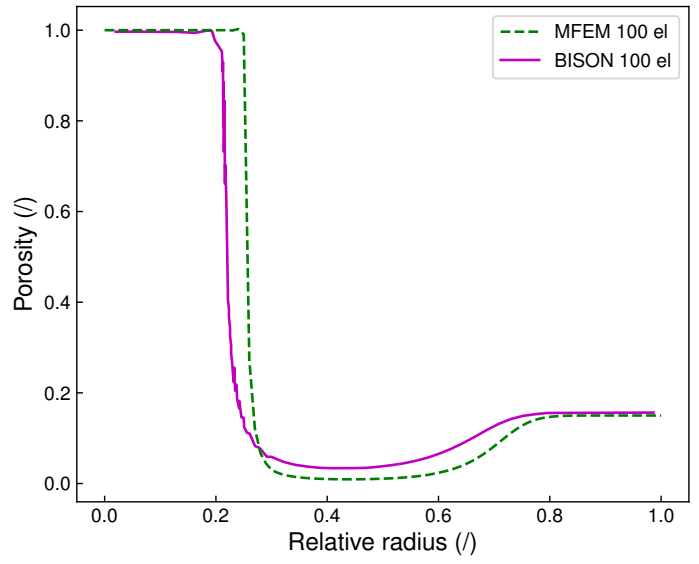

(b) 100 elements

Figure 6: Comparison of the results in terms of porosity as a function of the local radius obtained with MFEM and considering the SU stabilization technique to the one published in [14], using respectively 50 (Figure 6a) and 100 element (Figure 6b) meshes.

fuel pin irradiated in the Phenix sodium fast reactor, underlying the interaction and synergies of as-fabricated and crack-induced porosity.

\subsection{Analysis of the interaction between as-fabricated and crack-induced porosity}

In this work, we do not aim at directly accounting at the microscopic scale the physical phenomena governing the porosity transport due to the presence of cracks. Rather, we aim at demonstrating how such mechanisms could in principle be included in the present modeling framework. In particular, we seek a quantification of the contributions to the central hole formation arising from as-fabricated and crack-induced porosities. To qualitatively assess these mechanisms, we considered again the test-case analyzed in Section 4 in three different initial conditions:

a) A homogeneously dispersed porosity, with no cracks, accounting for a $15 \%$ void fraction (the same analyzed in Section 4.4);

b) A crack having a thickness such that the void fraction in the domain is equal to that of the first point;

c) A combination of the previous two, having a total porosity equal to the double of the previous cases.

The mesh considering the crack is a conforming mesh, constituted of triangular, first order elements. The crack pattern considered is a simplification of that developing in real conditions. The related assumptions are taken according to [37], i.e., only radial cracks developing under the first rise to power are considered, and axial and circumferential cracks are not accounted. The pellet is 
supposed to split in 8 fragments spanning 22.5 degrees in the angular direction. The crack thickness is calculated in order to reach the desired volumetric void (i.e., 15\%). The computational domain has the same radius and angular span as the one considered in the previous section. The mesh density varies along the radius and in the angular direction, to properly represent the interface between the crack and the fuel pellet. The meshes are reported in AppendixB.

In Figure 7 we report the initial condition of case b) in the upper half part of the figure and the solution at the end of simulation in the lower half part (reflected for the sake of representation). We can appreciate the migration of the porosity from the crack to the center of the pellet, originating the central hole. The crack healing (in the restructured zone) and concurrent central hole formation is coherent with experimental observation on irradiated $(\mathrm{U}, \mathrm{Pu}) \mathrm{O}_{2}$ fuel in sodium fast reactors $[1,19]$.

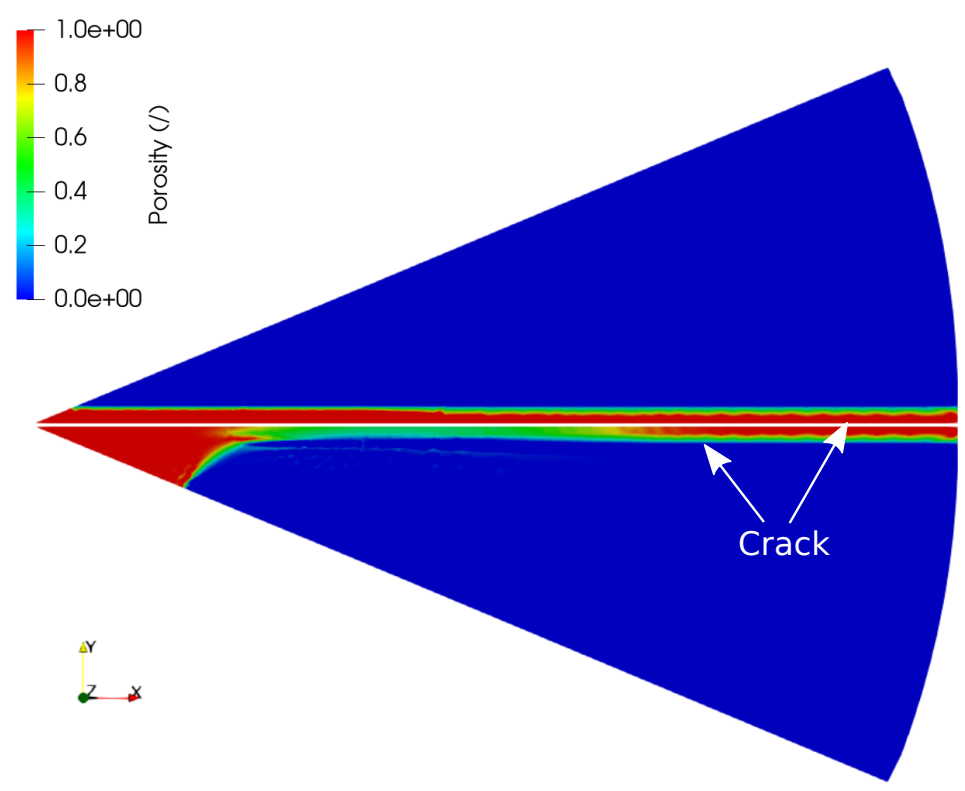

Figure 7: Initial condition (upper half) and final results (lower half) on the porosity distribution starting from a crack in the radial direction. For the sake of clarity, we underline that each circular sector represent a different condition.

The final configuration of cases a) and b) are reported in Figure 8. In this case, we are considering the same initial void fraction in the two circular sectors herein represented, but in the upper half, the initial porosity is homogeneously distributed in the volume, whereas in the lower half the initial porosity is distributed as in Figure 7. It can be seen that the resulting central holes have different radii, with the one obtained for the homogeneous porosity being larger.

Case c) is compared to case a) in Figure 9, with the former summing up to a 


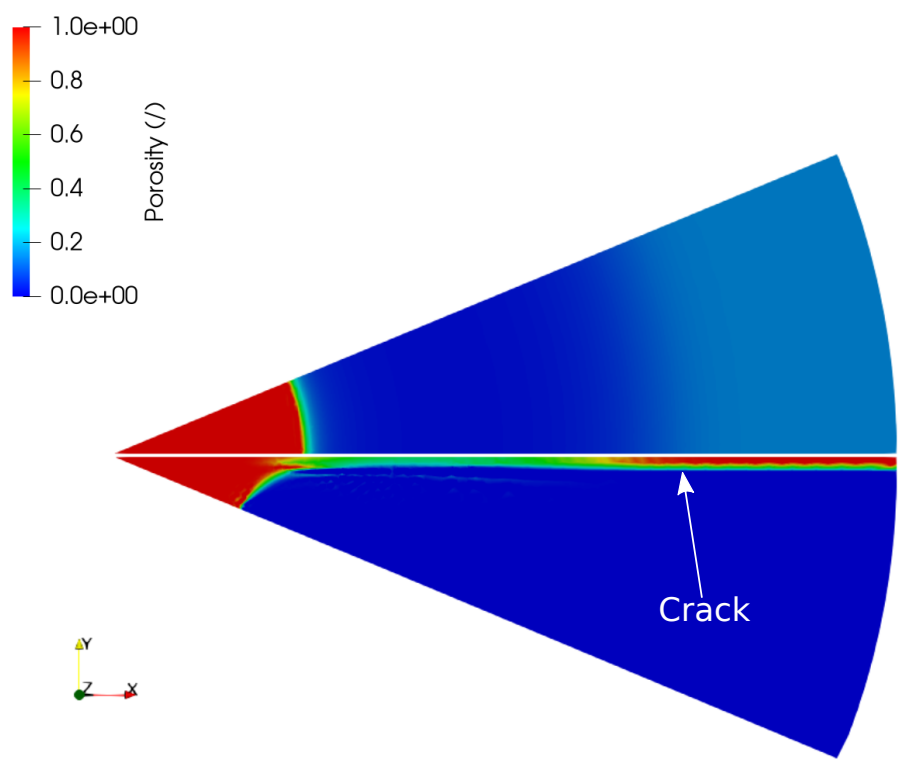

Figure 8: Final results on the porosity redistribution starting from the same volumetric void fraction distributed homogeneously in the domain (upper half) and in a crack (lower half). For the sake of clarity, we underline that each circular sector represents a separate case.

$30 \%$ of void fraction in the domain. This would be the situation occurring as the fuel is brought to power and undergoes cracking. In this case, the central hole results larger than in the aforementioned ones, due to the synergic contribution of the two porosity types. Yet, we can observe how the radius of the central void is less than sum of the individual contributions brought about considering the different porosities separately. This is expected and it is a direct consequence of the non-linear coupling between the equations governing temperature and porosity.

It is worth spending some comments on the results herein showed. First, the developed model has the original capability, compared to the state of the art, of computing the migration of crack-induced porosity through a direct representation of the crack itself and of its shape, rather than using an equivalent, homogenized porosity dispersed in the fuel. Second, the capability of correctly estimating the central hole extension is strictly dependent on the possibility of modeling the migration of crack-induced porosities, since it has been found that they can play a major role in determining its size [19]. In this sense, the model and the solvers developed in this work substantially surpass the state of the art capabilities of fuel performance codes. 


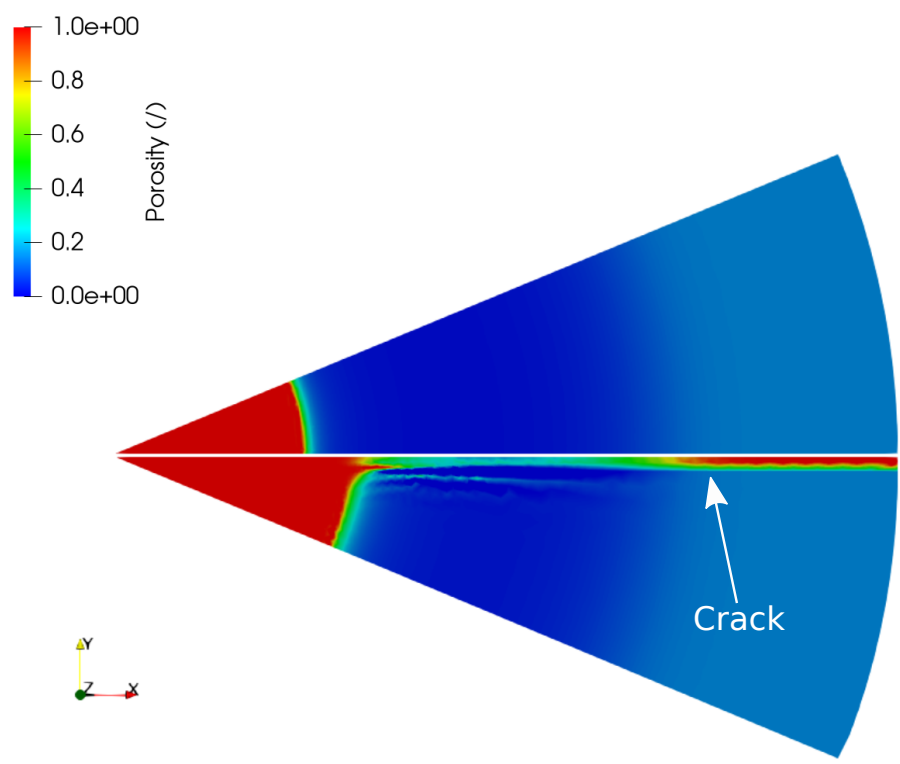

Figure 9: Final results on the porosity distribution, considering an initial condition having both as-fabricated and crack-induced porosity (lower half) and only as-fabricated porosity (upper half). For the sake of clarity, we underline that each circular sector represents a separate case.

\subsection{Preliminary assessment against experimental results}

The validation of the model developed in this work can be carried out only after its inclusion in the framework of a fuel performance code, since at the present status a large number of important phenomena governing the fuel behavior are not included in the modeling framework considered. Nonetheless, we present a preliminary assessment of the model against experimental data regarding the central hole formation. In particular, we analyze a fuel pellet included in a pin irradiated into the Phenix sodium fast reactor. The fuel pellet has an initial radius of $2.716 \mathrm{~mm}$ and an initial porosity of $4.1 \%$. Other details are reported in [19], in which the analysed case is referred to as "Fuel Pin 1". The choice of this pin is due to the "low" discharge burnup of the pin (around 1\% at.), which limits the impact of burnup-dependent phenomena (such as fuel swelling, constituents redistribution, or chemical speciation), not taken into account at the present moment by our model, on the final geometry of the fuel pellet. The goal of this exercise is to underline the importance of both as-fabricated and crack-induced porosity in determining the extent of fuel central void.

The setup of the case was carried out as follows. The fuel pellet experienced a linear heat rate around $400 \mathrm{~W} \mathrm{~cm}^{-1}$ throughout the whole irradiation. The fuel external temperature as a function of time was extracted from the 
PLEIADES/GERMINAL simulation of the pin presented in [19] and is reported in Figure 10. Two configurations are herein considered. The first one considers only the migration of as-fabricated porosity, thus the associated computational mesh consists of a circular sector spanning 22.5 degrees. The second one, which considers both as-fabricated and crack-induced porosity, has a geometry similar to that reported in the previous section, i.e., to the circular section representative for the fuel pellet is added a surface representative for the crack. The crack pattern is again those considered at the beginning of irradiation (e.g., see [37]) with straight, radial cracks. As for the crack thickness, we consider that the 8 fragments are fully displaced and in contact with the cladding, thus the initial internal void surface/volume due to the gap is conserved and transfered into the cracks $^{4}$ For both configurations, conforming and a priori refined first order triangular elements are used (see details in AppendixB).

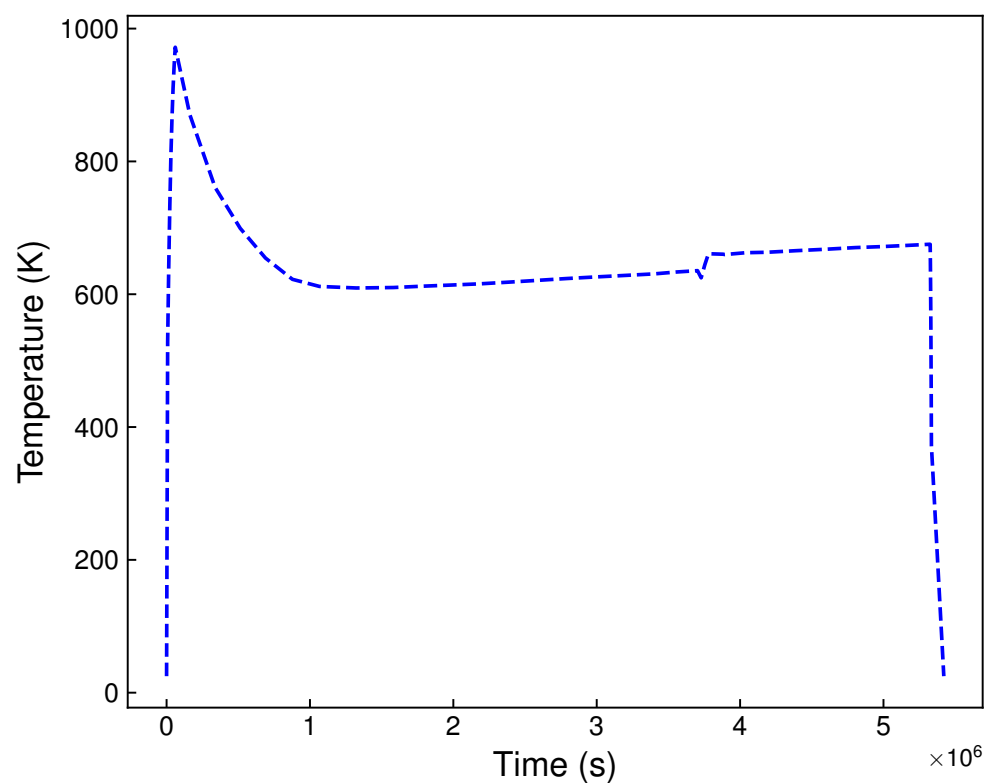

Figure 10: Fuel external temperature as a function of time for the fuel pellet considered in this assessment exercise.

The results at the end of the simulations are compared in Figure 11. As it is noticeable, the configuration considering the migration of the porosity induced by cracks $^{5}$ yields results that are closer to the experimental data then the one

\footnotetext{
${ }^{4}$ It is worth to notice that one can in principle choose a different number of fragments and a different angle to model the cracking pattern. In this case, the thickness of the crack would need to be adjusted to preserve the total free volume.

${ }^{5}$ The $1 \mathrm{D}$ plot reported here refers to a radius forming an angle with the $\mathrm{x}$ axis equal to 11
} 
considering only as-fabricated porosity. This result is in line with what was already shown in a previous work on the same [19] fuel pin, for which considering solely the migration of as-fabricated porosity was not enough to correctly assess the extent of the central void. As for the determination of the columnar grain region, the present model does not directly model its development, but can be associated to the part of the fuel pellet where the porosity is less than $2 \%$. If this value is considered as a threshold, the predictions by the present model are in line with the experimental data reported in Figure 11. The corresponding results on the entire computational domains are reported in Figure 12. Indeed, it must be underlined that this result is just a first assessment of the model capabilities, and that a more rigorous validation to a more consistent set of experimental data is needed to draw definitive conclusions. The analysis will be possible as the model will be included in a future version of the PLEIADES/GERMINAL fuel performance code.

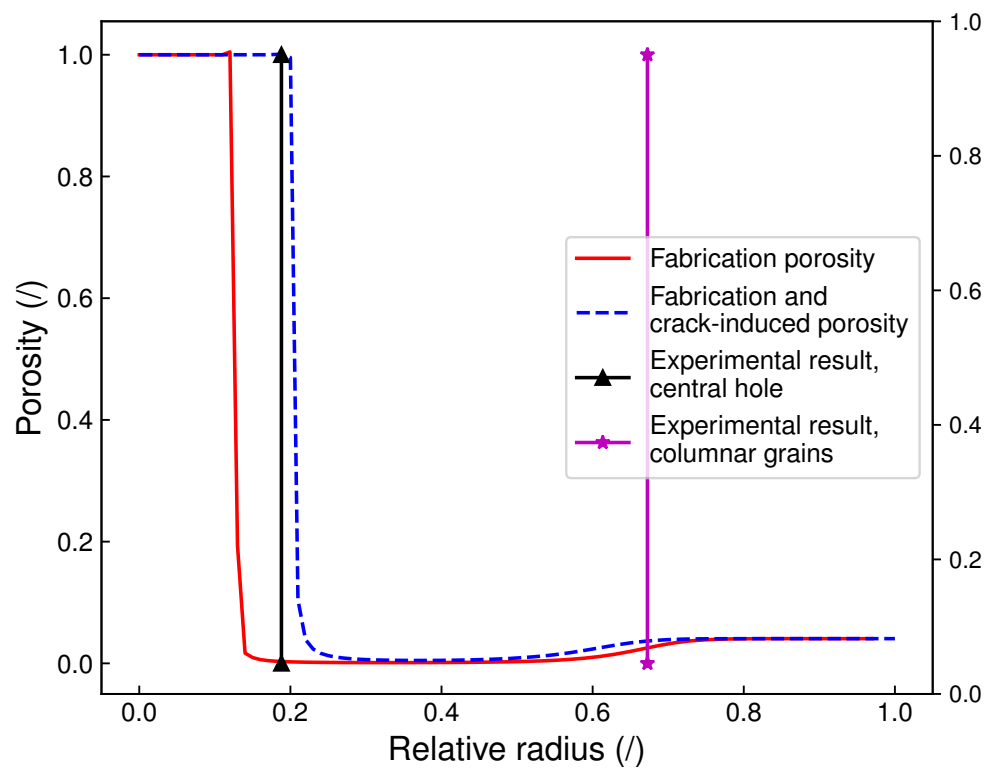

Figure 11: Comparison of experimental results to model predictions, considering only asfabricated porosity and including crack-induced porosity.

It is worth noticing that the underlying assumption is that we discard the mechanisms governing pores nucleation from cracks, and that the velocity expression employed to describe the migration of as-fabricated pores remains valid in this case. That is, we implicitly assume that pore are nucleated at the crack surface, driven by the circumferential component of the velocity, and then

degrees. 


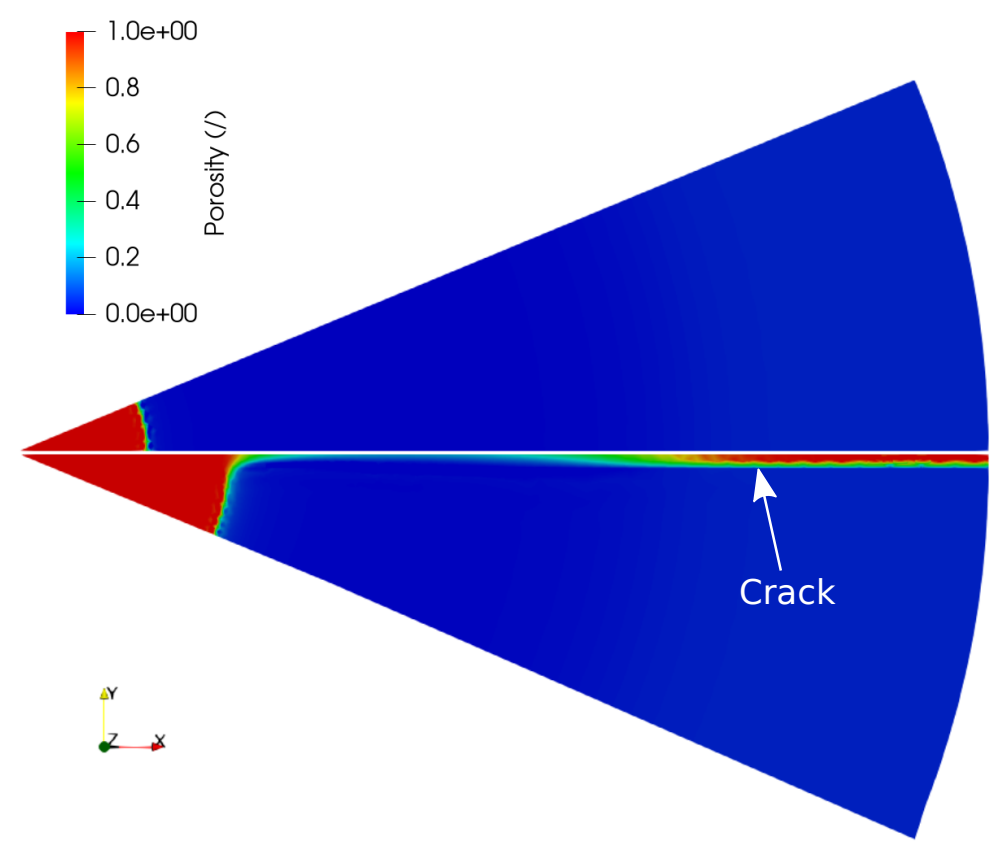

Figure 12: Contour plot of the porosity distribution in the two configurations, considering only as-fabricated porosity (upper half) and including crack-induced porosity (lower half). For the sake of clarity, we underline that each circular sector represent a different result.

transported in the circumferential and radial directions with the same velocity equation as the as-fabricated porosity. Under this modeling assumption, the pore velocity is also determining the rate at which crack-induced porosity is nucleated. A more realistically modeling framework would consider at the same time the evaporation of components from the "hot" segment of the crack, their diffusion in the vapor phase, and their subsequent condensation in the "cold" part. The inclusion of such phenomena in this framework is left as a future development of the present work. 


\section{Conclusions}

In this work, we proposed an original modeling framework for the coupled solution of the pore advection and heat conduction equations by the finite element method. The model is intended to describe the porosity migration in $(\mathrm{U}, \mathrm{Pu}) \mathrm{O}_{2}$ fuel irradiated in fast reactor conditions and considers the interdepencies among the solution variables and the parameters governing the problem. We implemented a numerical solver for the problem in MFEM, an open-source library for PDEs solution by the FEM. The solution algorithm includes a simple but robust fixed-point strategy combined to an explicit time solver. The modeling framework includes original and consistent stabilization techniques with respect to the state of the art in fuel performance codes for the pore advection equations, namely the Streamline-Upwind and Streamline-Upwind/Petrov-Galerkin techniques.

The developed finite element model has been compared to a model included in the fuel performance code BISON, based as well on the finite element method. The analysis was based on a test-case representative for the conditions experienced by a fuel pellet irradiated in a sodium fast reactor. The results obtained by our model, in terms of porosity distribution in the fuel pellet, are in agreement with those obtained by BISON. Moreover, the employed stabilization technique for the pore advection equation eliminates the spurious oscillations encountered in the BISON simulation when employing a coarse mesh, demonstrating the improvement brought about by the model developed in this work. Despite relying on the introduction of an "artificial" diffusion term, the proposed numerical framework enables us to introduce a mathematically consistent term in the equations, which is not inducing errors in the solution.

The model has been applied to the study of the porosity migration considering different types of porosity, namely including crack-induced alongside as-fabricated porosity. The analysis showed how the extension of the central void due to the migration of these different types of porosity is different, and combining the two types of porosity we demonstrate how the resulting central void is larger. This analysis is applied also to a fuel pin irradiated in the Phenix sodium fast reactor, and we underline the importance of considering crack-induced porosity in the assessment of the model against an experimental case.

Overall, the model we are presenting in this work, with respect to those available in the state of the art, includes on one hand a more rigorous, dedicated mathematical treatment of the spurious oscillations found in the solution of the pore advection equation in state-of-the-models utilizing the finite element method. On the other, accounting for crack-induced porosity stands out as a unique capability of the developed model with respect to the ones available in the open literature, and paves the way to its application in the study of crack healing in sodium fast reactor mixed oxides fuel.

Future developments of the outlined modeling framework encompass a reassessment of pore velocity and its study from a microscopic point of view, to derive a novel and robust behavioral law on this important parameter. More- 
over, the inclusion of the equations governing the plutonium, americium, and oxygen redistribution is of interest, in order to account for the effects of such quantities on the thermal and porosity solutions. Finally, once these modeling advancements will be available, we envisage a validation of the model against separate effect tests focused on the pore velocity model, e.g., comparing to the data from the Am-1 experiment [38], and a integral validation of the pore migration model, once it will be included in the GERMINAL/PLEIADES fuel performance code, against other Phenix irradiation data.

\section{Acknowledgments}

This work has been done in the framework of a cooperative program between CEA, FRAMATOME and EDF, devoted to the development of the fuel elements for GEN IV Reactors.

\section{AppendixA. SUPG solver verification}

To verify the correctness of the SU/SUPG implementation in MFEM, we compared the solutions obtained by our solvers against test-cases reported in the open literature as reference problems for the SUPG method development [22]. In particular, a steady-state case with a source term and a transient case with only the internal evolution are presented. The details about these testcases are reported in [22]. It is worth underlining that the implementation of the SU method does not call for a particular solver, since the term induced by this stabilization technique is a diffusion operator, whose discretization is already available in MFEM.

The results for the steady-state one are presented in Figure A.13. The testcase consists in considering a pure advection problem with constant advection velocity and a source term, which is reported in the figure. We consider a 1D mesh and impose a Dirichlet boundary condition at the inlet - i.e., at $x=0$. The results are in agreement with those proposed by Brooks and Hughes [22].

The test-case on the unsteady solution is taken again from Brooks and Hughes [22], and the results are reported in Figure A.14. The initial condition is a classic cosine hill, natural boundary conditions are enforced on the two ends of the 1D domain. A pure advection problem with a constant and unitary velocity oriented towards the positive $\mathrm{x}$ axis is considered. The time integration is carried out through an explicit Euler scheme, imposing a time step so that the Courant number is 0.5. As one can see, the solution obtained with our implementation is in line with that reported in the original paper for all the considered time steps. For the sake of comparison, the solution obtained by the SU stabilization technique is reported in Figure A.15, considering the same initial condition, geometry, and parameters. As one could see, the performance of the SU technique when considering a non-uniform initial condition is poorer than the SUPG. 


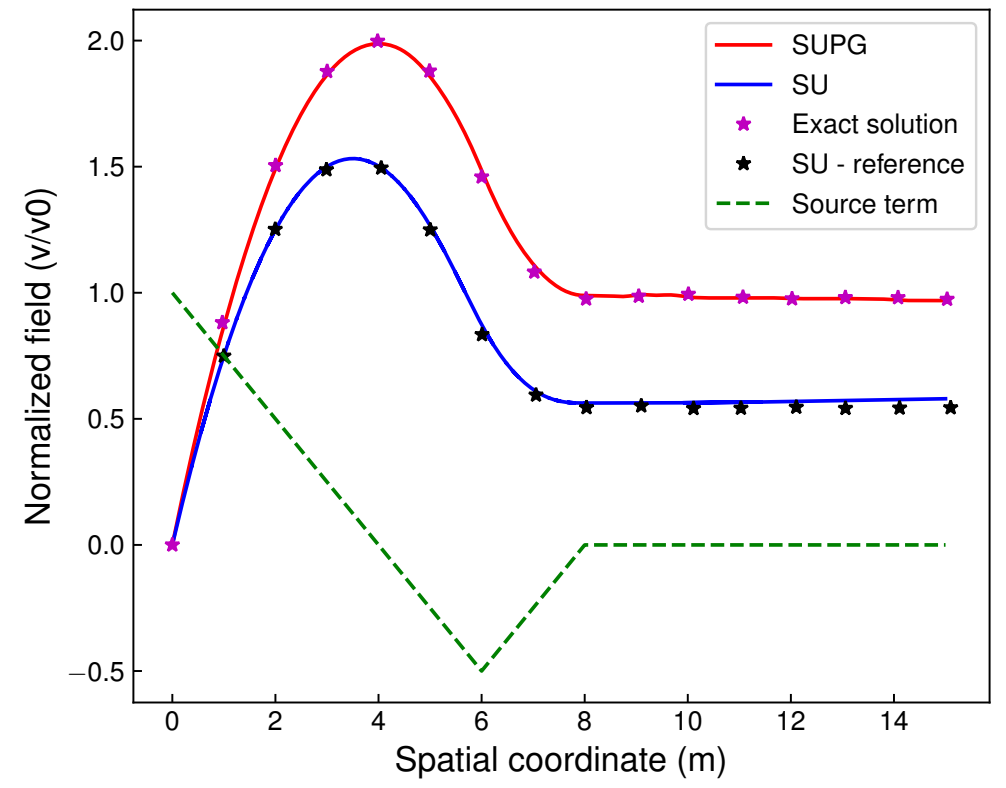

Figure A.13: Comparison of the reference and calculated solution employing SUPG techniques in the steady state test-case. The results obtained with the SU stabilization are also reported for the sake of comparison. 


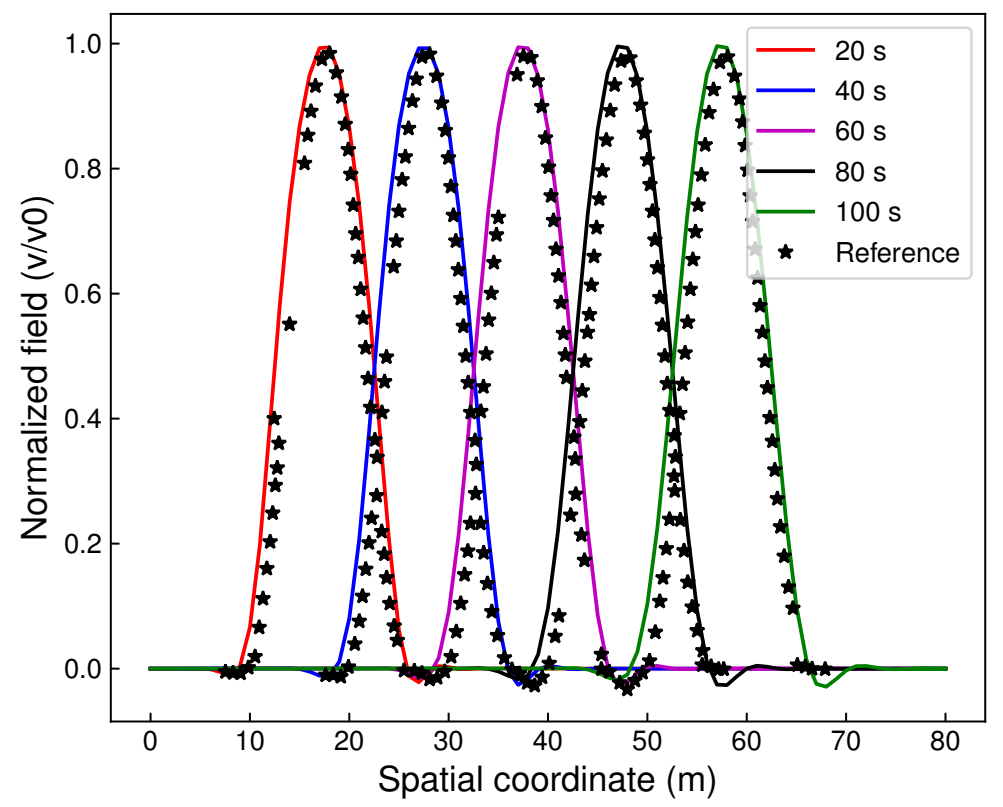

Figure A.14: Comparison of the reference solution (triangles) and calculated solution employing the SUPG stabilization after $20 \mathrm{~s}, 40 \mathrm{~s}, 60 \mathrm{~s}, 80 \mathrm{~s}$, and $100 \mathrm{~s}$. 


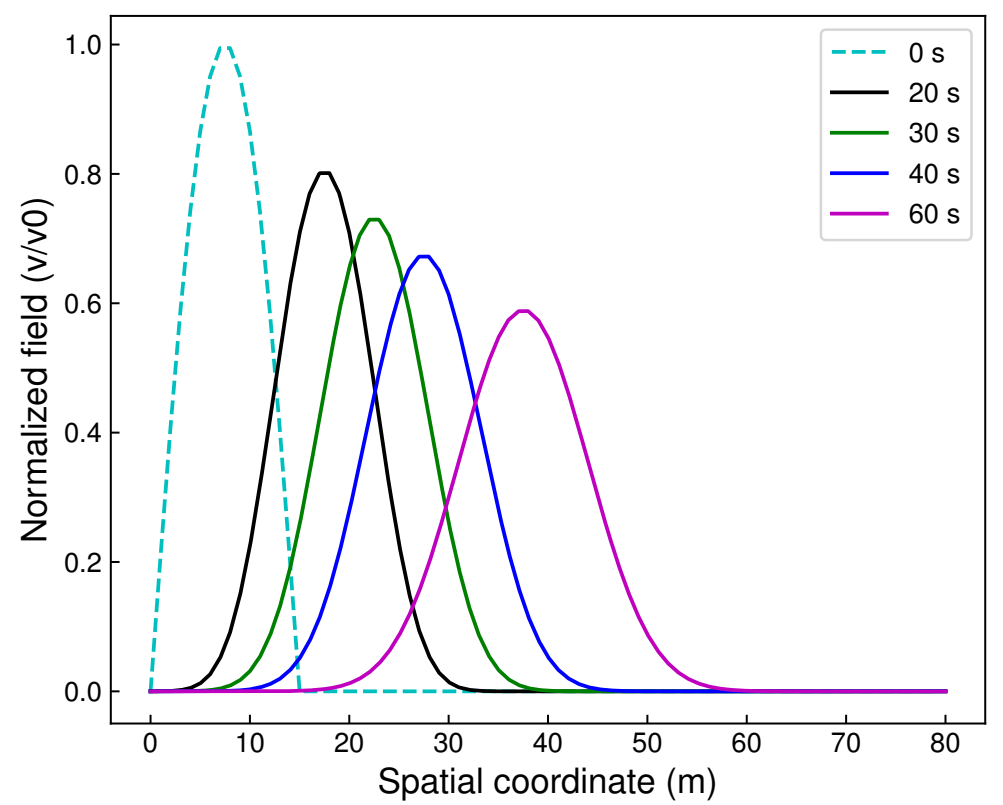

Figure A.15: Solution calculated on the test-case obtained considering an cosine-hill initial condition and employing the SU stabilization technique. 


\section{AppendixB. Computational meshes}

In this appendix, we report the computational meshes employed in this work. The non-conforming meshes employed in Sections 3 and 4, having 50 and 100 intervals in the radial directions, are reported in Figures B.16. The mesh is created such that the aspect ratio of the elements is as closest as possible to unity, and include a triangular central element.
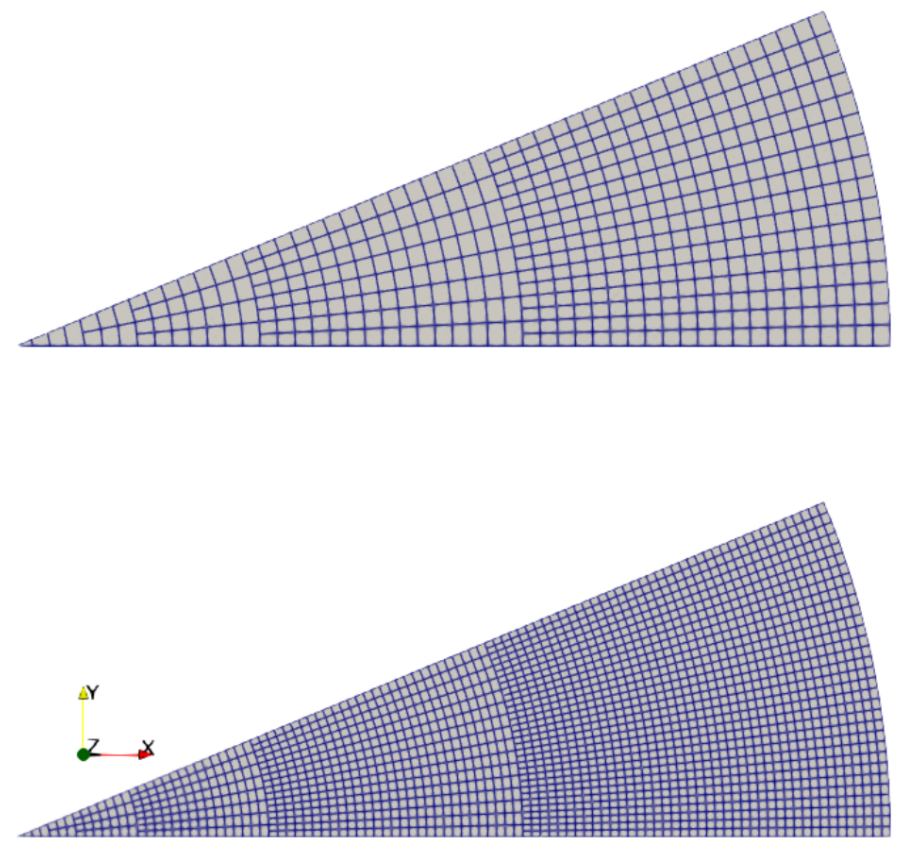

Figure B.16: Non conforming computational meshes having 50 and 100 intervals in the radial direction.

As for Section 5, different meshes are employed. In these cases, the mesh is conforming, and a priori refinement is applied in order to guarantee the solution is converged in mesh size. Hence, finer cells are employed at the central part of the pellet and close to the crack, where the steepest porosity gradients are expected. Triangular elements are employed in this case. An example of the mesh for the cracked domain is reported in Figure B.17, whereas the same mesh without the crack is the reported in Figure B.18. 


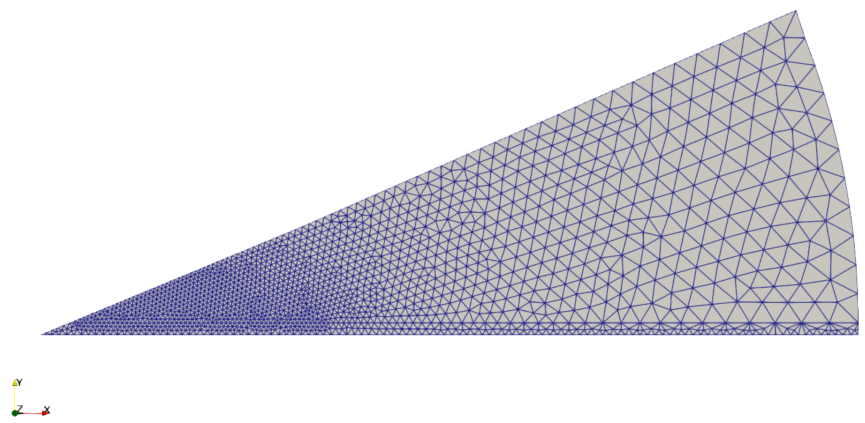

Figure B.17: Computational mesh for the cases including the crack.

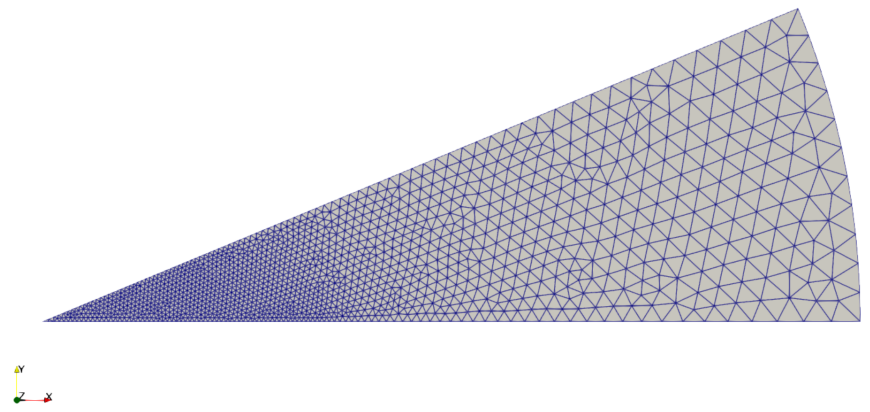

Figure B.18: Computational mesh for the cases having an un-cracked domain. 


\section{References}

[1] D. Olander, Fundamental aspects of nuclear reactor fuel elements, 1976. doi:10.1016/0022-3115(77)90226-4.

[2] M. Welland, Matter transport in fast reactor fuels, in: R. Konings, R. Stoller (Eds.), Comprehensive Nuclear Materials, Elsevier, 2020, pp. 630-676.

[3] M. Pelletier, Y. Guérin, Fuel Performance of Fast Spectrum Oxide Fuel, in: R. J. Konings, R. E. Stoller (Eds.), Comprehensive Nuclear Materials (Second Edition), Second Edition, Elsevier, Oxford, 2020, pp. 72-105. doi: https://doi.org/10.1016/B978-0-12-803581-8.11690-X.

[4] D. Staicu, Thermal Properties of Irradiated $\mathrm{UO}_{2}$ and MOX, in: R. J. Konings, R. E. Stoller (Eds.), Comprehensive Nuclear Materials (Second Edition), Second Edition, Elsevier, Oxford, 2020, pp. 149-172. doi : https : //doi.org/10.1016/B978-0-12-803581-8.11726-6.

[5] F. Nichols, Theory of columnar grain growth and central void formation in oxide fuel rods, Journal of Nuclear Materials 22 (2) (1967) 214-222. doi:https://doi.org/10.1016/0022-3115(67)90031-1.

[6] F. Nichols, Pore migration in ceramic fuel elements, Journal of $\mathrm{Nu}-$ clear Materials 27 (2) (1968) 137-146. doi:https://doi.org/10.1016/ 0022-3115(68) 90118-9.

[7] P. Sens, The kinetics of pore movement in $\mathrm{UO}_{2}$ fuel rods, Journal of Nuclear Materials 43 (3) (1972) 293-307.

[8] K. Lassmann, TRANSURANUS: a fuel rod analysis code ready for use, Journal of Nuclear Materials 188 (1992) 295-302.

[9] J. Melis, L. Roche, J. Piron, J. Truffert, GERMINAL — A computer code for predicting fuel pin behaviour, Journal of Nuclear Materials 188 (1992) 303-307. doi:https://doi.org/10.1016/0022-3115(92)90488-7.

[10] T. Ozawa, T. Abe, Development and Verifications of Fast Reactor Fuel Design Code CEPTAR, Nuclear Technology 156 (1) (2006) 39-55.

[11] A. Karahan, J. Buongiorno, Modeling of thermo-mechanical and irradiation behavior of mixed oxide fuel for sodium fast reactors, Journal of Nuclear Materials 396 (2) (2010) 272-282.

[12] S. E. Lemehov, F. Jutier, Y. Parthoens, B. Vos, S. Van Den Berghe, M. Verwerft, N. Nakae, MACROS benchmark calculations and analysis of fission gas release in MOX with high content of plutonium, Progress in Nuclear Energy 57 (2012) 117-124, nuclear Materials: Selected articles from the EMRS 2011 Spring Meeting. doi:https://doi.org/10.1016/j.pnucene. 2011.12.010. 
[13] T. Uwaba, T. Mizuno, J. Nemoto, I. Ishitani, M. Ito, Development of a mixed oxide fuel pin performance analysis code "CEDAR": Models and analyses of fuel pin irradiation behavior, Nuclear Engineering and Design 280 (2014) 27-36. doi:https://doi.org/10.1016/j.nucengdes. 2014. 08.032 .

[14] S. Novascone, P. Medvedev, J. W. Peterson, Y. Zhang, J. Hales, Modeling porosity migration in LWR and fast reactor MOX fuel using the finite element method, Journal of Nuclear Materials 508 (2018) 226-236.

[15] M. Lainet, B. Michel, J.-C. Dumas, M. Pelletier, I. Ramière, GERMINAL, a fuel performance code of the PLEIADES platform to simulate the in-pile behaviour of mixed oxide fuel pins for sodium-cooled fast reactors, Journal of Nuclear Materials 516 (2019) 30-53. doi:https://doi.org/10.1016/ j.jnucmat.2018.12.030.

[16] Y. Sukjai, K. Shirvan, Enhancing FRAPCON fuel performance code for physical phenomena at high temperature and high burnup, Journal of $\mathrm{Nu}-$ clear Materials 517 (2019) 113-127.

[17] T. Ozawa, S. Hirooka, M. Kato, S. Novascone, P. Medvedev, Development of fuel performance analysis code, BISON for MOX, named Okami: Analyses of pore migration behavior to affect the MA-bearing MOX fuel restructuring, Journal of Nuclear Materials 553 (2021) 153038. doi: https: //doi.org/10.1016/j.jnucmat.2021.153038.

[18] L. Luzzi, T. Barani, B. Boer, L. Cognini, A. Del Nevo, M. Lainet, S. Lemehov, A. Magni, V. Marelle, B. Michel, D. Pizzocri, A. Schubert, P. Van Uffelen, M. Bertolus, Assessment of three European fuel performance codes against the SUPERFACT-1 fast reactor irradiation experiment, $\mathrm{Nu}-$ clear Engineering and Technologydoi:https://doi.org/10.1016/j.net. 2021.04 .010 .

[19] M. Temmar, B. Michel, I. Ramiere, N. Favrie, Multi-physics modelling of the pellet-to-cladding gap closure phenomenon for SFR fuel performance codes, Journal of Nuclear Materials 529 (2020) 151909. doi:https://doi. $\mathrm{org} / 10.1016 / \mathrm{j}$. jnucmat. 2019.151909.

[20] A. Quarteroni, Numerical Models for Differential Problems, MS\&A, Springer Milan, 2010.

[21] K. Bathe, Finite Element Procedures, Prentice Hall, Englewood Cliffs, NJ, 1996.

[22] A. Brooks, T. Hughes, Streamline upwind/Petrov-Galerkin formulations for convection dominated flows with particular emphasis on the incompressible Navier-Stokes equations, Computer Methods in Applied Mechanics and Engineering 32 (1) (1982) 199-259. 
[23] R. Anderson, J. Andrej, A. Barker, J. Bramwell, J.-S. Camier, J. Cerveny, V. Dobrev, Y. Dudouit, A. Fisher, T. Kolev, W. Pazner, M. Stowell, V. Tomov, I. Akkerman, J. Dahm, D. Medina, S. Zampini, MFEM: A modular finite element methods library, Computers \& Mathematics with Applications 81 (2021) 42-74. doi:https://doi.org/10.1016/j. camwa.2020.06.009.

[24] D. Knoll, D. Keyes, Jacobian-free Newton-Krylov methods: a survey of approaches and applications, Journal of Computational Physics 193 (2) (2004) 357-397. doi:https://doi.org/10.1016/j.jcp.2003.08.010. URL https://www.sciencedirect.com/science/article/pii/ S0021999103004340

[25] F. Nichols, Kinetics of diffusional motion of pores in solids: A review, Journal of Nuclear Materials 30 (1) (1969) 143-165.

[26] R. Codina, Comparison of some finite element methods for solving the diffusion-convection-reaction equation, Computational methods in applied mechanics and engineering 156 (1998) 185-210.

[27] V. Di Marcello, V. Rondinella, A. Schubert, J. van de Laar, P. Van Uffelen, Modelling actinide redistribution in mixed oxide fuel for sodium fast reactors, Progress in Nuclear Energy 72 (2014) 83-90, symposium E @ E-MRS 2013 SPRING MEETING Scientific basis of the nuclear fuel cycle. doi:https://doi.org/10.1016/j.pnucene.2013.10.008.

URL https://www.sciencedirect.com/science/article/pii/ S0149197013002011

[28] W. J. Lackey, F. J. Homan, A. R. Olsen, Porosity and actinide redistribution during irradiation of $(\mathrm{u}, \mathrm{pu})_{\mathrm{O}_{2}}$, Nuclear Technology 16 (1) (1972) $120-142$.

[29] S. Guarro, D. Olander, Actinide redistribution due to pore migration in hypostoichiometric mixed-oxide fuel pins, Journal of Nuclear Materials 57 (2) (1975) 136-144. doi:https://doi.org/10.1016/0022-3115(75) 90253-6.

URL https://www.sciencedirect.com/science/article/pii/ 0022311575902536

[30] C. Clement, Analytic solutions to mass transport equations for cylindrical nuclear fuel elements, Journal of Nuclear Materials 68 (1) (1977) 54-62. doi:https://doi.org/10.1016/0022-3115(77)90216-1. URL https://www.sciencedirect.com/science/article/pii/ 0022311577902161

[31] C. Clement, M. Finnis, Plutonium redistribution in mixed oxide (U, $\mathrm{Pu}) \mathrm{O}_{2}$ nuclear fuel elements, Journal of Nuclear Materials 75 (1) (1978) 193-200. doi:https://doi.org/10.1016/0022-3115(78)90044-2. URL https://www.sciencedirect.com/science/article/pii/ 0022311578900442 
[32] M. Kato, K. Maeda, T. Ozawa, M. Kashimura, Y. Kihara, Physical properties and irradiation behavior analysis of Np- and Am-Bearing MOX Fuels, Journal of Nuclear Science and Technology 48 (4) (2011) 646-653. doi:10.1080/18811248.2011.9711745.

[33] A. Eucken, Allgemeine gesetzmäßigkeiten für das wärmeleitvermögen verschiedener stoffarten und aggregatzustände, Forschung auf dem Gebiet des Ingenieurwesens A 11 (1940) 6-20.

[34] A. Magni, T. Barani, A. Del Nevo, D. Pizzocri, D. Staicu, P. Van Uffelen, L. Luzzi, Modelling and assessment of thermal conductivity and melting behaviour of mox fuel for fast reactor applications, Journal of Nuclear Materials 541 (2020) 152410. doi:https://doi.org/10.1016/j.jnucmat.2020.152410. URL https://www.sciencedirect.com/science/article/pii/ S0022311520310187

[35] D. R. O'Boyle, R. O. Meyer, Redistribution of Uranium and Plutonium in mixed-oxide fuels during irradiation, in: Panel Meeting on the Behaviour and Chemical State of Fission Products in Irradiated Fuels, IAEA, Vienna, Austria, 1972, pp. 41-66.

[36] D. R. Olander, The kinetics of actinide redistribution by vapor migration in mixed oxide fuels (I). By cracks, Journal of Nuclear Materials $49(1973 / 74)$ $21-34$.

[37] J. Sercombe, B. Michel, C. Riglet-Martial, O. Fandeur, Modeling of Pellet Cladding Interaction, in: R. J. Konings, R. E. Stoller (Eds.), Comprehensive Nuclear Materials (Second Edition), 2nd Edition, Elsevier, Oxford, 2020, pp. 417-465.

[38] K. Tanaka, S. Miwa, S. ichi Sekine, H. Yoshimochi, H. Obayashi, S. ichi Koyama, Restructuring and redistribution of actinides in Am-MOX fuel during the first $24 \mathrm{~h}$ of irradiation, Journal of Nuclear Materials 440 (1) (2013) 480-488. doi:https://doi.org/10.1016/j.jnucmat.2013.01. 351. 